Probl. Anal. Issues Anal. Vol. 5 (23), No. 2, 2016, pp. 33-37

DOI: $10.15393 /$ j3.art.2016.3330

UDC 517.54

A. P. KOPYLOV

\title{
QUASI-ISOMETRIC MAPPINGS AND THE $p$-MODULI OF PATH FAMILIES
}

\begin{abstract}
In this article, we study a connection between quasiisometric mappings of $n$-dimensional domains and the $p$-moduli of path families. In particular, we obtain explicit (and sharp) estimates for the distortion of the $p$-moduli of path families under $K$-quasi-isometric mappings.
\end{abstract}

Key words: $p$-modulus of path families, p-capacity of the condenser, quasi-isometric mappings

\section{Mathematical Subject Classification: 30C65}

1. Introduction. The article is devoted to the study of problems connected with the search for a complete descritpion of quasi-isometric mappings of $n$-dimensional domains in terms of the $p$-moduli of families of paths (curves). Note that this problem (for quasi-isometric mappings and also for quasiconformal mappings, space mappings with bounded distortion, mappings with finite distortion, homeomorphisms with finite mean dilatations, mappings with $(p, q)$-distortion etc) was successfully solved by many mathematicians (see, for example, [1]-[3]; see also [4]-[9]). Our main goal is to obtain explicit (and sharp) estimates for the distortion of the $p$-moduli of families of paths and curves under $K$-quasi-isometric mappings. Here we use the following, metric definition of such mappings:

Definition 1. Let $K \in\left[1, \infty\left[\right.\right.$. A homeomorphism $f: U_{1} \rightarrow U_{2}$ of domains $U_{1}$ and $U_{2}$ in $\mathbb{R}^{n}$ is called $K$-quasi-isometric if

$$
K^{-1} \leq \liminf _{y \rightarrow x} \frac{|f(y)-f(x)|}{|y-x|} \leq \limsup _{y \rightarrow x} \frac{|f(y)-f(x)|}{|y-x|} \leq K
$$

for any $x \in U_{1}$. A homeomorphism $f: U_{1} \rightarrow U_{2}$ is called quasi-isometric if it is $K$-quasi-isometric for some $K \in[1, \infty[$. 
A. P. Kopylov

Our main result is

Theorem 1. Suppose that $f: U_{1} \rightarrow U_{2}$ is a $K$-quasi-isometric homeomorphism of bounded domains $U_{1}$ and $U_{2}$ in $\mathbb{R}^{n}$, where $n \geq 2(1 \leq K<$ $<\infty)$. Then

$$
K^{2-p-n} M_{p}(\Gamma) \leq M_{p}(f(\Gamma)) \leq K^{p+n-2} M_{p}(\Gamma)
$$

for every $p \in] 1, \infty\left[\right.$ and any family $\Gamma$ of paths $\gamma$ such that $\operatorname{Im} \gamma \subset \operatorname{cl} U_{1}$.

Remark 1. The quantity $M_{p}(\Gamma)$, where $1 \leq p<\infty$, is called the $p$ modulus of the path family $\Gamma$ and defined as

$$
M_{p}(\Gamma)=\inf _{\rho \in \mathcal{R}(\Gamma)} \int_{\mathbb{R}^{n}}[\rho(x)]^{p} d x,
$$

where $\mathcal{R}(\Gamma)$ is the set of all nonnegative Borel measurable functions $\rho$ : $\mathbb{R}^{n} \rightarrow \dot{\mathbb{R}}$ such that $\int_{\gamma} \rho d s \geq 1$ for every rectifiable path $\gamma \in \Gamma$.

It should be noted that our main result (Theorem 1) is conceptually most close to the results on quasi-isometries in [1.

For example, using Theorem 1 in [10] and our result, Corollary 3 to Theorem $4.4^{\prime}$ in [1, Chapter 5 , Section 4 , can be supplemented by the following assertion:

Theorem 2. Under the conditions of Theorem 1 ,

$$
K^{2-p-n} C_{p}^{1}\left(F_{0}, F_{1} ; U_{1}\right) \leq C_{p}^{1}\left(f\left(F_{0}\right), f\left(F_{1}\right) ; U_{2}\right) \leq K^{p+n-2} C_{p}^{1}\left(F_{1}, F_{2} ; U_{1}\right)
$$

for every $p \in] 1, \infty\left[\right.$ and any condenser $\left(F_{0}, F_{1} ; U\right)$.

Remark 2. $C_{p}^{1}\left(F_{0}, F_{1}, U\right)$ is the p-capacity of the condenser $\left(F_{0}, F_{1} ; U\right)$ $\left(F_{0}\right.$ and $F_{1}$ are closed disjoint nonempty sets in cl $U$, where $U \subset \widetilde{\mathbb{R}}^{n}=$ $=\mathbb{R}^{n} \cup\{\infty\}$ is an open set), i.e.,

$$
C_{p}^{1}\left(F_{0}, F_{1} ; U\right)=\inf \int_{U}|\nabla u|^{p} d x,
$$

where infimum is taken over all functions $u \in C^{\infty}(U) \cap L_{p}^{1}(U)$ that are equal to unity (zero) in some neighborhood of $F_{0}\left(F_{1}\right)$ (see [1] ).

In what follows, for $x \in \mathbb{R}^{n}$ and $E \subset \mathbb{R}^{n}$, $\operatorname{dist}(x, E)=\inf _{y \in E}|x-y|$, all paths $\gamma:[\alpha, \beta] \rightarrow \mathbb{R}^{n}$, where $\alpha, \beta \in \mathbb{R}$, are assumed continuous and non-constant, and $l(\gamma)$ means the length of a path $\gamma$. 
2. Proof of Theorem 1. The proof of Theorem 1 follows the lines of the proof of the second claim of Theorem 6.5 in $[12$.

Let $\Gamma$ be a family of paths in the domain $U_{1}$ (i.e., of paths $\gamma:[a, b] \rightarrow$ $\rightarrow \mathbb{R}^{n}$ such that $\left.\operatorname{Im} \gamma \subset \operatorname{cl} U_{1}\right)$. Consider the subfamily $\Gamma^{*}$ of $\Gamma$ consisting of all locally rectifiable paths $\gamma \in \Gamma$ such that $f$ is absolutely continuous on every closed subpath of $\gamma$. Since $f$ is a quasi-isometry, $f \in A C L_{p}$ for all $p>1$ (see, for example, [13, 12, for the definition of the class $\left.A C L_{p}\right)$; therefore, $M_{p}\left(\Gamma_{0}\right)=0$ for the family $\Gamma_{0}$ of all locally rectifiable paths in $U_{1}$ having subpaths on which the mapping $f$ is not absolutely continuous ([13]). The fact that $\Gamma \backslash \Gamma^{*} \subset \Gamma_{0}$ and the properties of moduli imply the equality $M_{p}\left(\Gamma \backslash \Gamma^{*}\right)=0$. Consequently, $M_{p}\left(\Gamma^{*}\right)=M_{p}(\Gamma)$. Therefore, for proving, for example, the left-hand inequality in (1), which we will do below, it suffices to show that $M_{p}\left(\Gamma^{*}\right) \leq K^{p+n-2} M_{p}(f(\Gamma))$, where $f(\Gamma)=\{f \circ \gamma: \gamma \in \Gamma\}$.

Let $E$ be a Borel subset in $U_{1}$ that contains all points $x \in U_{1}$ at which $f$ is not differentiable and all those points $x$ in $U_{1}$ at which $f$ is differentiable but the Jacobian $J(x, f)=0$, moreover, mes $E\left(=\operatorname{mes}_{n} E\right)=0$. Here we use the facts that a quasi-isometric mapping is quasiconformal and the set of points of nondegenerate differentiability of a quasiconformal mapping is a set of full measure with respect to its domain of definition.

Assume that $\widetilde{\rho} \in \mathcal{R}\left(f\left(\Gamma^{*}\right)\right)\left(f\left(\Gamma^{*}\right)=\left\{f \circ \gamma: \gamma \in \Gamma^{*}\right\}\right)$, i.e., $\int_{\widetilde{\gamma}} \widetilde{\rho}(x) d s \geq$ $\geq 1$ for every locally rectifiable path $\widetilde{\gamma} \in f\left(\Gamma^{*}\right)$. Define a function $\rho: \mathbb{R}^{n} \rightarrow$ $\rightarrow \mathbb{R}^{n}$ by setting $\rho(x)=\widetilde{\rho}(f(x))\left\|f^{\prime}(x)\right\|$ if $x \in U_{1} \backslash E, \rho(x)=\infty$ if $x \in E$, and $\rho(x)=0$ if $x \in \mathbb{R}^{n} \backslash U_{1}$. Arguing as in the proof of the second part of Theorem 6.5 in [12] (or of Theorem 32.3 in [14], which is the $n$-dimensional variant of the first theorem), we further infer that $\rho \in \mathcal{R}\left(\Gamma^{*}\right)$, and hence

$$
\begin{gathered}
M_{p}(\Gamma)=M_{p}\left(\Gamma^{*}\right) \leq \int_{\mathbb{R}^{n}} \rho^{p} d x=\int_{U_{1}}[\widetilde{\rho}(f(x))]^{p} \| f^{\prime}(x)||^{p} d x= \\
=\int_{U_{1}}[\widetilde{\rho}(f(x))]^{p} \frac{\| f^{\prime}(x)||^{p}}{|J(x, f)|}|J(x, f)| d x \leq K^{p+n-2} \int_{U_{1}}[\widetilde{\rho}(f(x))]^{p}|J(x, f)| d x= \\
=K^{p+n-2} \int_{U_{2}}[\widetilde{\rho}(y)]^{p} d y=K^{p+n-2} \int_{\mathbb{R}^{n}}[\widetilde{\rho}(y)]^{p} d y .
\end{gathered}
$$

In (2), we have used the fact that, since $f$ is $K$-quasi-isometry, it is easy to verify the inequality $\frac{\| f^{\prime}(x)||^{p}}{|J(x, f)|} \leq K^{p+n-2}$ for $x \in U_{1} \backslash E$. Taking 2 
into account and recalling that the inverse mapping $f^{-1}$ is also $K$-quasiisometric, we finally get 11 .

3. Sharpness of estimates (1). Suppose that $\left.\Pi_{n}=\right] 0,1\left[^{n}, K \in\right.$ $\in[1, \infty[$, and

$$
f: x=\left(x_{1}, \ldots, x_{n-1}, x_{n}\right) \mapsto\left(K x_{1}, \ldots, K x_{n}, K^{-1} x_{n}\right), \quad x \in \Pi_{n} .
$$

Then $f: \Pi_{n} \rightarrow f\left(\Pi_{n}\right)$ is a $K$-quasi-isometric homeomorphism, and if $p \in] 1, \infty[$ and $\Gamma$ is the family of paths joining the sets $] 0,1\left[^{n-1} \times\{0\}\right.$ and ] $0,1\left[{ }^{n-1} \times\{1\}\right.$ in $\Pi_{n}, f(\Gamma)=\{f \circ \gamma: \gamma \in \Gamma\}$ then $M_{p}(\Gamma)=1$, and

$$
M_{p}(f(\Gamma))=\frac{K^{n-1}}{\left(K^{-1}\right)^{p-1}}=K^{p+n-2} .
$$

Thus, the rightmost estimate in (1) is sharp. Similarly, the leftmost estimate is also sharp.

Remark 3. It is worth noting that estimates (1) were previously unknown.

Acknowledgment. The author was partially supported by the Russian Foundation for Basic Research (Grant 11-01-00819-a), the Interdisciplinary Project of the Siberian and Far-Eastern Divisions of the Russian Academy of Sciences (2012-2014 no. 56), the State Maintenance Program for the Leading Scientific Schools of the Russian Federation (Grant NSh921.2012.1) and the Exchange Program between the Russian and Polish Academies of Sciences (Project 2014-2016).

\section{References}

[1] Gol'dshtein V. M., Reshetnyak Yu. G. Introduction to the theory of functions with generalized derivatives, and quasiconformal mappings. Moscow: "Nauka", 1983, (in Russian). Rev. engl. transl.: Quasiconformal mappings and Sobolev spaces. Dordrecht etc.: Kluwer Academic Publishers, 1990 (Mathematics and Its Applications: Soviet Series, 54).

[2] Gehring F. W. Lipschitz mappings and the p-capacity of rings in n-space. Advances in the theory of Riemann surfaces (Proc. Conf., Stony Brook, N.Y., 1969), Ann. of Math. Studies, No. 66. Princeton, N. J.: Princeton Univ. Press, 1971, pp. 175-193.

[3] Golberg A. Quasiisometry from different points of view. J. Math. Sci. (N. Y.), 2014, vol. 196, no. 5, pp. 617-631. DOI: 10.1007/s10958-014-1680-y 
[4] Martio O., Ryazanov V., Srebro U., Yakubov E. Moduli in Modern Mapping Theory. New York: Springer Science + Business Media, LLC, 2009. DOI: $10.1007 / 978-0-387-85588-2$

[5] Martio O., Ryazanov V., Srebro U., Yakubov E. Mappings with finite length distortion. J. Anal. Math., 2004, vol. 93, pp. 215-236. DOI: 10.1007/BF02789308

[6] Salimov R. R., Sevost'yanov E. A. The Poletskii and Väisälä inequalities for the mappings with $(p, q)$-distortion. Complex Variables and Elliptic Equations, 2014, vol. 59, no. 2, pp. 217-231. DOI: 10.1080/17476933.2012.731397

[7] Salimov R. R., Sevost'yanov E. A. ACL and differentiability of open discrete ring ( $p, Q)$-mappings. Matematychni studii, 2011, vol. 35, no. 1, pp. 28-36.

[8] Sevost'yanov E. A. The Väisälä inequality for mappings with finite length distortion. Complex Variables and Elliptic Equations, 2010, vol. 55, no. 13, pp. 91-101. DOI: 10.1080/17476930902998993

[9] Salimov R. R., Sevost'yanov E. A. ACL and differentiability of the open discrete ring mappings. Complex Variables and Elliptic Equations, 2010, vol. 55, no. 1-3, pp. 49-59. DOI: 10.1080/17476930902999009

[10] Shlyk V. A. The equality between p-capacity and p-modulus. Sib. Math. J., 1993, vol. 34, no. 6, pp. 1196-1200. DOI: 10.1007/BF00973485

[11] Maz'ya V. G. Sobolev spaces. (Prostranstva S. L. Soboleva.) Leningrad: Izdatel'stvo Leningradskogo Universiteta, 1985 (in Russian). Engl. transl.: Sobolev spaces. Berlin etc.: Springer-Verlag, 1985.

[12] Väisälä J. On quasiconformal mappings in space. Ann. Acad. Sci. Fenn., Ser. A I, 1961, no. 298, pp. 1-36.

[13] Fuglede B. Extremal length and functional completion. Acta Math., 1957, vol. 98, no. 1, pp. 171-219. DOI: 10.1007/BF02404474

[14] Väisälä J. Lectures on n-Dimensional Quasiconformal Mappings. BerlinHeidelberg-New York: Springer-Verlag, 1971. DOI: 10.1007/BFb0061216

Received October 05, 2016.

In revised form, December 05, 2016.

Accepted December 02, 2016.

Sobolev Institute of Mathematics

4, Acad. Koptyuga pr., Novosibirsk 630090, Russia;

Novosibirsk State University

2, Pirogova st., Novosibirsk 630090, Russia

E-mail: apkopylov@yahoo.com 